Regionally Extinct and 175 as threatened; of the reptiles two species are categorized as Regionally Extinct and 136 as threatened; of the birds three species are categorized as Regionally Extinct and 146 as threatened; and of the mammals 169 are categorized as threatened. The main threats to China's vertebrates are human activities, habitat loss and overexploitation. For the mammals, overexploitation is the main threat, with 116 mammal species affected, followed by human interference and habitat loss.

ZHIGANG JIANG Key Laboratory of Animal Ecology and Conservation Biology, Institute of Zoology, Chinese Academy of Sciences, Beijing, China. E-mail jiangzg@ioz.ac.cn

\section{A little-known blackbuck population in Chennai's suburban forests}

The blackbuck or Indian antelope Antilope cervicapra is a medium-sized ungulate now endemic to India (it is Regionally Extinct in Bangladesh and Pakistan). It has been placed under Schedule I of the Wildlife (Protection) Act, 1972, and is categorized as Near Threatened on the IUCN Red List.

Blackbuck formerly occurred across almost the whole of the Indian subcontinent but have disappeared from many areas as a result of habitat destruction through conversion to agricultural use.

In Tamil Nadu, the southernmost state in which blackbuck survive in the wild, there are a few widely separated populations. An additional group of blackbuck was discovered recently by $\mathrm{KN}$ in Chennai's suburban forests, in Thaiyur Reserve Land, c. $40 \mathrm{~km}$ from Tambaram, in Kanchipuram District. One adult male, one adult female and four juveniles were discovered in July 2014. In a subsequent visit three adult males, nine adult females, 11 juveniles and three fawns were seen.

Discussions with local people indicated that 200-250 blackbuck may inhabit this $8 \mathrm{~km}^{2}$ degraded area. Further research is required within and around the Reserve Land to assess the population's size. Blackbuck inhabit grasslands that are relatively open, with short grass, and avoid thick cover. In Tamil the blackbuck is called veli maan (antelope of the open areas). The species is well adapted to semi-desert habitat and can tolerate heat and drought. Thaiyur Reserve Land is, however, threatened by urbanization and neighbouring industries, and the traffic on the nearby ThaiyurKelambakkam road is a direct threat to blackbuck. There is a need for immediate action from the appropriate authorities to take the necessary steps to conserve this population of blackbuck, which is one of the few thriving populations of the species outside protected areas in Tamil Nadu. This is also an opportunity: Thaiyur Reserve Land and the blackbuck could serve as an ecology laboratory for local students and could be used to educate the suburban public about conservation.

KAnNADASAN NARASIMMARAJAN and MANU ThOMAS Mathai Department of Zoology, Madras Christian College, Tambaram, Chennai, India. E-mail wildlife9protect@gmail.com

\section{The rare Kolar leaf-nosed bat}

India is home to 117 species of bats, of which only two species are included in the Wildlife Protection Act (1972). Bats in India are subjected to great pressures: they are hunted and eaten for their supposed medicinal properties, their roosting sites are marauded and burnt because of superstitious beliefs, and their habitats are destroyed to accommodate the ever-growing human population and other development activities.

The Endangered Kolar leaf-nosed bat Hipposideros hypophyllus is endemic to Kolar district, Karnataka, and is known from only two localities: Hanumanhalli and Therahalli. Since its description in 1994 this species had not been sighted and its conservation status was unknown. With the help of funding from The Mohamed bin Zayed Species Conservation Fund we conducted two surveys (during November-December 2013 and April-May 2014), to locate roosts and to conduct nocturnal acoustic monitoring, in both localities. We located a subterranean cave, the type locality of the species, on a granite hill in Hanumanhalli village, and were successful in mist-netting the species there. We estimate that this population may number no more than 200 individuals. We found the species sharing its roost with five other species of bats. In Therahalli, although we located a subterranean cave and other potential roost sites, we were not successful in finding the species. The cave at Hanumanhalli is threatened by illegal granitemining activities in the immediate vicinity. Two other roost sites that we found on the same hill had been abandoned by bats.

Based on our findings, we recommend that the Red List category of the Kolar leaf-nosed bat is changed from Endangered to Critically Endangered. As there is only one known roost site, urgent steps need to be taken to halt nearby mining and quarrying activities. Failing this, the species will inevitably become extinct in the near future. Following a discussion with the Divisional Forest Officer of Kolar district, stone quarrying activity in the vicinity of the subterranean cave at Hanumanhalli has been halted, and the matter is now under further discussion with the appropriate authorities.

C. SRINIVASUlu Natural History Museum and Wildlife Biology \& Taxonomy Lab, Department of Zoology, Osmania University, Hyderabad, Telangana, India, and Zoo 